Check for updates

Cite this: J. Mater. Chem. C, 2018, 6,6460

Received 17th April 2018, Accepted 29th May 2018

DOI: $10.1039 / c 8 t c 01809 j$

rsc.li/materials-c

\section{Thermally stable resistive switching of a polyvinyl alcohol-based atomic switch $\dagger$}

\author{
Karthik Krishnan, ${ }^{\text {ab }}$ Masakazu Aono ${ }^{a}$ and Tohru Tsuruoka (D) *a
}

\begin{abstract}
We demonstrate thermally stable resistive switching of a solid polymer electrolyte (SPE)-based atomic switch device, in which a Ag salt incorporated polyvinyl alcohol (PVA) film is sandwiched between Ag and Pt electrodes. The observed switching is attributed to the formation and dissolution of a Ag filament in the PVA matrix. The device exhibits good endurance characteristics, with large ON/OFF ratios $\left(>10^{5}\right)$ in the temperature range between 25 and $70{ }^{\circ} \mathrm{C}$. This temperature stability can be explained by competition between increased segmental mobility and water evaporation at higher temperatures. The results indicate that the thermal behavior of the polymer matrix plays a crucial role in determining the temperature window for stable operation of SPE-based atomic switches.
\end{abstract}

\section{Introduction}

Owing to a variety of advantages such as good film forming ability, easy scalability, high adhesiveness on various substrates, and low fabrication costs, polymer materials are considered to be essential candidates to revolutionize not only the field of energy conversion and storage, but also the field of memory, logic, and neuromorphic device applications. ${ }^{1-6}$ Redox-based resistive switching in a metal-insulator-metal (MIM) structure, in which a solid polymer electrolyte (SPE) film is sandwiched between an electrochemically active metal electrode ( $\mathrm{Ag}$ or $\mathrm{Cu}$ ) and an inert metal electrode ( $\mathrm{Pt}$ or $\mathrm{Au}$ ), is a promising approach for the development of next-generation organic memory devices. ${ }^{7}$ In SPE-based memory devices, the observed resistive switching has mostly been explained by the redox-induced formation and rupture of conducting filaments in the polymer matrix. ${ }^{8,9}$ The application of forward bias (relative to the electrochemically active electrode) induces oxidation of metal atoms at the active electrode, and the oxidized ions transport toward the inert electrode. Then, precipitation of metal atoms on the inert electrode takes place and its growth to the active electrode forms a metal filament between the electrodes. Subsequent reverse bias disconnects the filament by the opposite redox reactions at the thinnest part. Thus, by sweeping bias voltage, the device resistance switches between a high-resistance (OFF) state and a low-resistance $(\mathrm{ON})$ state. From the similarity of its operating

\footnotetext{
${ }^{a}$ International Center for Materials Nanoarchitectonics (WPI-MANA), National Institute for Materials Science, 1-1 Namiki, Tsukuba 305-0044, Japan. E-mail: TSURUOKA.Tohru@nims.go.jp

${ }^{b}$ Council of Scientific and Industrial Research, Central Electrochemical Research Institute, Karaikudi 630-003, India. E-mail: karthikk@cecri.res.in

$\dagger$ Electronic supplementary information (ESI) available. See DOI: 10.1039/ c8tc01809j
}

mechanism to the "gap-type atomic switch," whose resistance across a nanometer gap between a mixed conductor electrode and an inert electrode is switched between OFF and ON states under bias sweeping, ${ }^{10}$ the SPE-based, MIM-structured device is referred to as a "gapless-type atomic switch". ${ }^{11}$

In recent years, we have demonstrated resistive switching operations in a MIM-structured device using a Ag salt incorporated polyethylene oxide (Ag-PEO) as the SPE. PEO is a highly crystalline polymer that possesses high ionic conductivity at room temperature, which is well above the glass transition temperature $T_{\mathrm{g}}$. The device showed not only nonvolatile resistive switching behaviour but also conductance quantization under bias sweeping. ${ }^{12,13}$ The filament morphology was found to be determined by the redox rates at the metal/SPE interfaces and by ion mobility in the PEO matrix. ${ }^{14}$ However, when the substrate temperature was increased from 25 to $75{ }^{\circ} \mathrm{C}$, the operation voltages significantly decreased in magnitude and the switching behaviour changed from nonvolatile to volatile. ${ }^{15}$ This can be explained by the increased conductivity of $\mathrm{Ag}$ ions in the amorphous PEO-salt complex region of the polymer matrix at elevated temperatures. The results suggest that the stability in device performance seems to be determined by the intrinsic thermal property of the polymer matrix. However, to date, few reports are available on the impact of the thermal properties of the polymer matrix on the resistive switching characteristics of polymer-based memory devices. Recently, Zhang et al. reported thermally stable resistive switching, using poly(triphenylamine), based on the formation of cationic conducting filamentary pathways. ${ }^{16}$

Here, we report thermally stable resistive switching operations in polyvinyl alcohol (PVA)-based atomic switches. PVA is a hydrophilic linear polymer with excellent thermal and mechanical stability and is widely reported to facilitate the enhancement of 
ion conducting properties for various applications. ${ }^{17-22}$ Due to the strong interaction between polymer chains and water molecules, PVA showed higher conductivity even at temperatures lower than $T_{\mathrm{g} \cdot}{ }^{23,24}$ Here, we report resistive switching behaviour of a Ag/Agsalt included PVA (Ag-PVA)/Pt device in a wide temperature widow ranging from 25 to $70{ }^{\circ} \mathrm{C}$.

\section{Experimental}

MIM-structured Ag/Ag-PVA/Pt devices were fabricated on $\mathrm{Si}$ substrates covered with $300 \mathrm{~nm}$ thick $\mathrm{SiO}_{2}$, in which a spincoated Ag-PVA film with a salt concentration of $3 \mathrm{wt} \%$ is sandwiched between electron-beam (EB) deposited $\mathrm{Ag}$ and $\mathrm{Pt}$ electrodes, as illustrated schematically in the inset of Fig. 1a. By optimizing the spin coating conditions, a uniform Ag-PVA film with a thickness of $\sim 100 \mathrm{~nm}$ was formed on the Pt electrode. The device had a cross-point structure with a junction area of $5 \times 5 \mu^{2}$. Current-voltage $(I-V)$ measurements were performed in atmosphere using a two-probe system equipped with a heater stage and a semiconductor characterization system. The substrate temperature was varied between 25 and $70{ }^{\circ} \mathrm{C}$. The thermal behaviour of the Ag-PVA electrolyte was evaluated by means of differential scanning calorimetry (DSC) and thermal gravity (TG) analysis. Details of the device fabrication and the experimental methods employed are provided in the ESI. $\dagger$
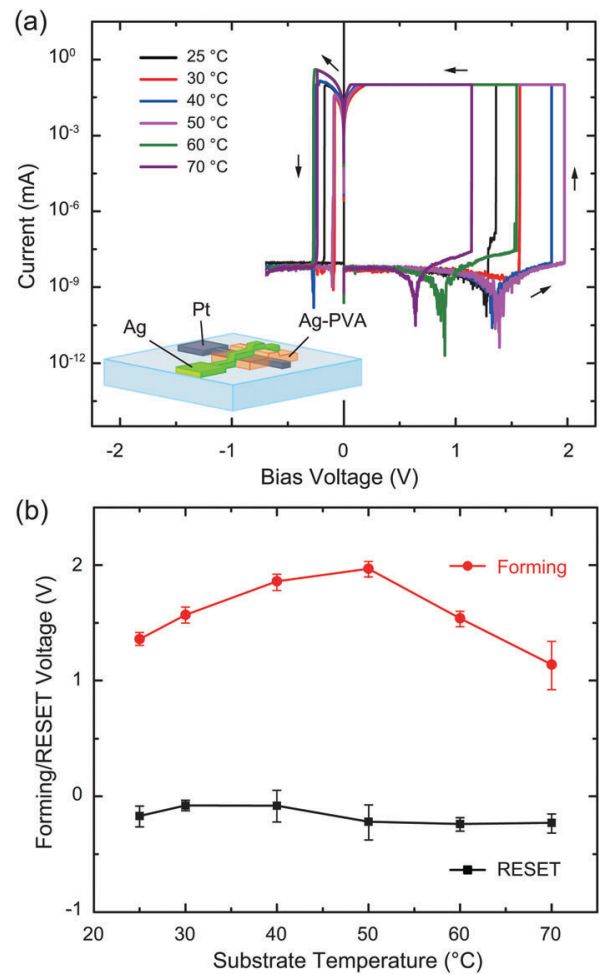

Fig. 1 (a) Forming characteristics of a Ag/Ag-PVA/Pt device, measured for substrate temperatures ranging from 25 to $70{ }^{\circ} \mathrm{C}$. The inset illustrates the fabricated device. (b) The forming voltage and subsequent RESET voltage plotted as a function of the substrate temperature, evaluated from the I-V data of 4-7 devices at each temperature.

\section{Results and discussion}

Fig. 1a shows the typical $I-V$ curves measured at the first sweep cycle for different substrate temperatures. The bias voltage was swept relative to the $\mathrm{Ag}$ electrode with a constant sweep rate of $7.2 \mathrm{mV} \mathrm{s}^{-1}$, while the Pt electrode was grounded. For all the measured temperatures, the device exhibited a SET operation from the pristine OFF state to the ON state under forward bias and a RESET operation from the ON state to the OFF state under reverse bias, showing bipolar resistive switching behaviour. This first SET operation corresponds to the first formation of a metal filament between the $\mathrm{Ag}$ and Pt electrodes. This is referred to as the forming process. Fig. 1b plots the forming voltage and the subsequent RESET voltage, evaluated from the $I-V$ data of 4-7 devices at each temperature. As the substrate temperature was increased from $25{ }^{\circ} \mathrm{C}$, the forming voltage (the voltage of the first SET operation) initially increased from $\sim 1.4 \mathrm{~V}$ to $\sim 2 \mathrm{~V}$ up to $50{ }^{\circ} \mathrm{C}$, and then decreased to $\sim 1.1 \mathrm{~V}$ at $70{ }^{\circ} \mathrm{C}$. The RESET voltage seemed to increase slightly in magnitude with the increase of substrate temperature, but it is difficult to determine any clear tendency.

After the forming process, the Ag/Ag-PVA/Pt device exhibited repeatable resistive switching behaviour in the temperature range observed. Fig. $2 \mathrm{a}$ and b represent the typical $I-V$ curves for $10^{2}$ consecutive sweep cycles, measured with the same sweep rate at 25 and $70{ }^{\circ} \mathrm{C}$, respectively. Although the SET and RESET voltages fluctuated somewhat, the device exhibited stable switching behaviour at both substrate temperatures, as indicated by the black (first sweep) and red $\left(10^{2}\right.$ th sweep) curves. Fig. 3a plots
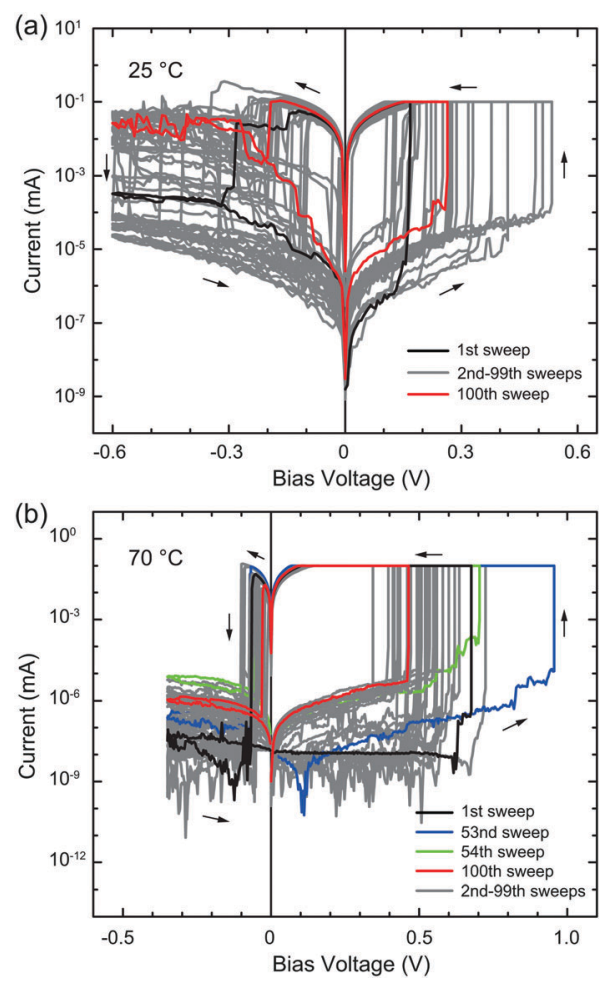

Fig. 2 Resistive switching characteristics of a Ag/Ag-PVA/Pt device, measured at 25 (a) and $70{ }^{\circ} \mathrm{C}$ (b) with a constant sweep rate of $7.2 \mathrm{mV} \mathrm{s}^{-1}$ and $I_{\mathrm{CC}}$ of $100 \mu \mathrm{A}$ for $10^{2}$ continuous sweep cycles. 

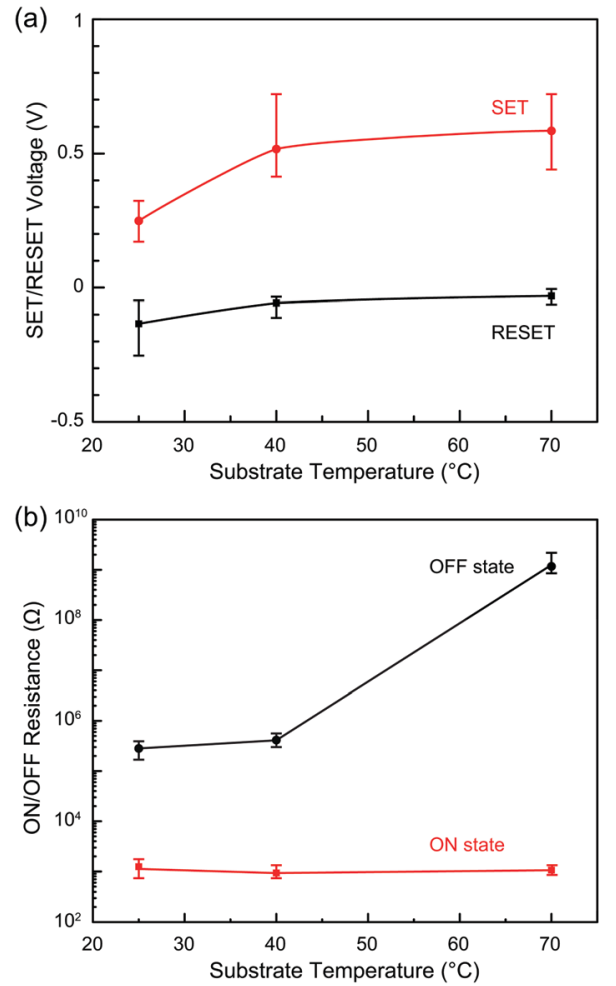

Fig. 3 SET/RESET voltages (a) and ON/OFF resistances (b) plotted as a function of the substrate temperature.

the SET and RESET voltages as a function of the substrate temperature, evaluated from the $I-V$ data for selected temperatures $\left(25,40\right.$, and $\left.70{ }^{\circ} \mathrm{C}\right)$. The SET and RESET voltages were approximately 0.27 and $-0.13 \mathrm{~V}$ at $25{ }^{\circ} \mathrm{C}$, respectively. As the substrate temperature increased, the SET voltage rose to $\sim 0.5 \mathrm{~V}$ up to $40{ }^{\circ} \mathrm{C}$ and then increased gradually to $\sim 0.6 \mathrm{~V}$ at $70{ }^{\circ} \mathrm{C}$. The magnitude of the RESET voltage decreased from $-0.13 \mathrm{~V}$ (at $25{ }^{\circ} \mathrm{C}$ ) to $-0.03 \mathrm{~V}$ (at $70{ }^{\circ} \mathrm{C}$ ). The SET voltages were much smaller than the forming voltages. At the first sweep cycle, more $\mathrm{Ag}$ ions are required for the formation of a metal filament, giving rise to a large forming voltage. After the second sweep cycle, most parts of the filament remained in the polymer matrix, and the formation and dissolution take place in a small gap of the filament, resulting in smaller SET voltages. This situation has already been reported for planar $\mathrm{Ag} / \mathrm{Ag}-\mathrm{PEO} / \mathrm{Pt}$ devices $^{14}$ and was also confirmed for planar Ag/Ag-PVA/Pt devices (not shown here).

The corresponding temperature variation of the $\mathrm{ON}$ and $\mathrm{OFF}$ resistances is shown in Fig. 3b. The ON resistance was kept on the order of $10^{3} \Omega$. This means that the size of the formed metal filament is similar for the temperature range observed. In contrast, the OFF resistance remained on the order of $10^{5} \Omega$ up to $40{ }^{\circ} \mathrm{C}$ and then increased to $\sim 10^{9} \Omega$ at $70{ }^{\circ} \mathrm{C}$, which suggests a drastic decrease in the conductivity of the OFF state at higher temperatures. It was found that the ON states (with $\sim 10^{3} \Omega$ ) can be retained for at least more than $1 \mathrm{~h}$, while the OFF states show longer retention times (i.e. on the order of days).
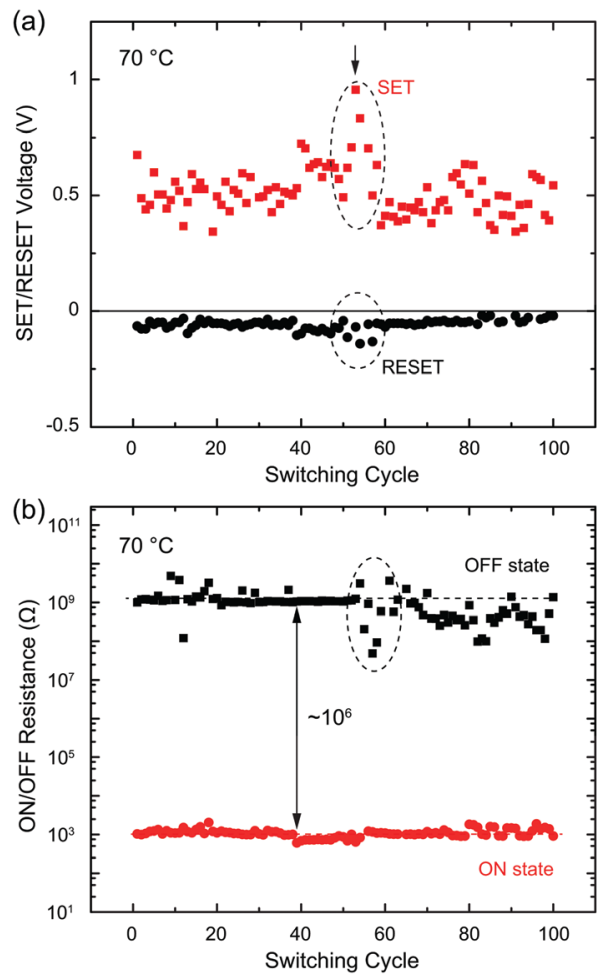

Fig. 4 Example of the endurance characteristics of a Ag/Ag-PVA/Pt device, measured at $70{ }^{\circ} \mathrm{C}$. SET/RESET voltages (a) and ON/OFF resistances (b), plotted as a function of the switching (sweep) cycle.

Note that if a pure PVA film is used as the electrolyte matrix, the forming and subsequent resistive switching are not observed, despite the existence of the electrochemically active $\mathrm{Ag}$ electrode. Only when Ag salt is incorporated into the PVA matrix can one see repeatable resistive switching. This result means that both the Ag ions dissolved in the PVA matrix and oxidized from the Ag electrode participate in the formation of a metal filament, which is similar to what occurs in a Ag/Ag-PEO/ Pt device. ${ }^{8,14}$

Fig. $4 \mathrm{a}$ and $\mathrm{b}$ plot the SET/RESET voltages and the corresponding $\mathrm{ON} / \mathrm{OFF}$ resistances at $70{ }^{\circ} \mathrm{C}$ as a function of the sweep (switching) cycle, respectively, extracted from the $I-V$ curves of Fig. 2b. The SET voltages are distributed mostly between 0.3 and $0.7 \mathrm{~V}$, while the RESET voltages range from -0.1 to $-0.02 \mathrm{~V}$. The corresponding $\mathrm{ON}$ and OFF resistances were on the order of $10^{3}$ and $10^{9} \Omega$, respectively, up to $\sim 50$ th sweep cycle, resulting in an $\mathrm{ON} / \mathrm{OFF}$ resistance ratio of $\sim 10^{6}$. After $\sim 60$ th sweep cycle, the OFF resistance decreased slightly, with some fluctuation, but the ON resistance remained on the order of $\sim 10^{3} \Omega$. As a result, the ON/OFF resistance ratio was greater than $10^{5}$ even at the $10^{2}$ th switching cycle.

The current minima in the $I-V$ curves measured at $70{ }^{\circ} \mathrm{C}$ are significantly distributed over the cycle-to-cycle, as seen in Fig. $2 \mathrm{~b}$. This current minimum in the logarithmic scale corresponds to a zero-crossing point in a linear scale, at which the corresponding voltage is associated with electric polarization and an open-circuit voltage. $^{25,26}$ In our device, electric polarization is generated by redox reactions at the metal/PVA interfaces and charge distributions in the 
PVA matrix, resulting in charge accumulation of cations and anions in the opposite electrode interfaces. We also see that electric polarization dynamically changes during switching cycles. For example, at the 53rd sweep cycle, the device was turned on with a large SET voltage, as shown by the blue curve in Fig. $2 \mathrm{~b}$ and by the arrow in Fig. $4 \mathrm{a}$. After the subsequent RESET operation, electric polarization almost disappeared at the next (54th) sweep cycle, but the OFF current increased (OFF resistance decreased) by about one order of magnitude, as shown by the green curve in Fig. 2b. We can see that the SET/RESET voltages and the OFF resistance fluctuate in several sweep cycles around the 53rd cycle, as indicated by the dotted circles in Fig. 4a and b. After that, the OFF resistance continued to fluctuate up to the $10^{2}$ th cycle. This result indicates that control of charge distribution in the polymer matrix is critically important in achieving stable switching behaviour.

To reveal the origin of the stable switching at higher temperatures, the thermal properties of the Ag-PVA electrolyte were investigated by DSC/TG analysis. Fig. 5 shows a typical DSC and TG curves measured for a thick Ag-PVA film with the same Ag salt concentration. They were obtained at the first scan with the same heating rate, in nitrogen atmosphere. Three characteristic features appeared in the DSC curve. First, a baseline shift appears at around $40{ }^{\circ} \mathrm{C}$, in which a cross point between tangents on the lower temperature side and on the slope corresponds to $T_{\mathrm{g}}$ of the amorphous region. From the obtained curve, $T_{\mathrm{g}}$ of the Ag-PVA electrolyte is estimated to be $37.5{ }^{\circ} \mathrm{C}$. Second, a large endotherm peak is observed at around $199{ }^{\circ} \mathrm{C}$, which corresponds to the melting point $T_{\mathrm{m}}$ of the crystalline region. The observed $T_{\mathrm{g}}$ and $T_{\mathrm{m}}$ are lower than those of pure (and dry) PVA (80 and $227{ }^{\circ} \mathrm{C}$, respectively). ${ }^{27}$ These lowered values are due to the plasticizing effect of the Ag salt and water incorporated in the PVA matrix. Finally, an additional broad endotherm is observed at around $125{ }^{\circ} \mathrm{C}$. If the DSC curve was re-measured immediately after the curve of Fig. 5 in nitrogen atmosphere (corresponding to the second scan), this endotherm disappeared and $T_{\mathrm{g}}$ increased to $73.2{ }^{\circ} \mathrm{C}$, as shown in Fig. S1 of ESI. $\dagger$ However, if the film was left in air, the endotherm developed again and $T_{\mathrm{g}}$ was lowered to below $40{ }^{\circ} \mathrm{C}$. Thus, this third endotherm is attributed to evaporation of water from the PVA matrix. ${ }^{28,29}$ Similar behaviour was also observed in other polymer materials such as polyvinyl pyrrolidone. ${ }^{30}$

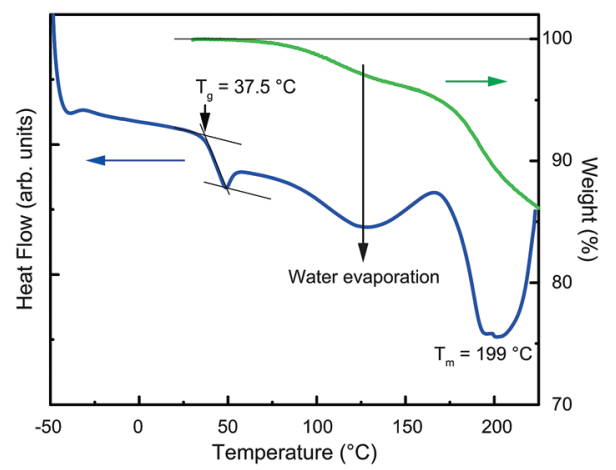

Fig. 5 DSC and TG curves measured for Ag-PVA electrolyte with a Ag salt concentration of $3 \mathrm{wt} \%$.
From the DSC curve in Fig. 5, the degree of crystallinity of the Ag-PVA electrolyte is estimated to be $47 \%$, which is much lower than that of the Ag-PEO electrolyte (78\%) with the same Ag salt concentration. ${ }^{15}$ The TG curve indicates that water starts to evaporate from the Ag-PVA film above $\sim 50{ }^{\circ} \mathrm{C}$, as evidenced by the observed weight loss. ${ }^{31}$ This thermal behaviour of the Ag-PVA electrolyte can explain the observed switching characteristics. Under ambient conditions, water absorption into the Ag-PVA film leads to the formation of hydrogen bonds between PVA hydroxyl groups and water molecules. Hence, water acts as a plasticizer by an increase of the free volume in the amorphous phase, ${ }^{32}$ and $T_{\mathrm{g}}$ is considerably lowered. As a result, the film changes from a glassy state to a rubbery state, which reduces the resistance of the film and increases ionic conductivity. Therefore, because of the increased ionic conductivity, the Ag/Ag-PVA/Pt device exhibits resistive switching behaviour even at $25{ }^{\circ} \mathrm{C}$, which is well below $T_{\mathrm{g}}$ of pure (and dry) PVA. As the substrate temperature increases, molecular segmental mobility increases, further enhancing ionic conductivity. However, a portion of the absorbed water is evaporated from the PVA matrix, which decreases the interaction between hydroxyl groups and water molecules. This retards conductivity because of decreased free volume and thus increases the OFF resistance at higher temperatures, as observed experimentally (Fig. 2). Due to the competition between the increased segmental mobility and the decreased interaction between hydroxyl groups and water, stable SET voltages can be realized at higher temperatures. It is expected that the water evaporation should start from a lower temperature, because the Ag-PVA film of the device is much thinner than the film used for the DSC/TG analysis.

Compared with the SET voltage, the RESET voltage exhibited different temperature behaviour; it decreased in magnitude with increased substrate temperature. This behaviour can be explained by the temperature stability of the filament formed. Because the ON resistance is fixed by the current compliance function at approximately $10^{3} \Omega$ (Fig. $3 \mathrm{~b}$ ), the size of the filament is expected to be similar for all the temperatures. Under these circumstances, the filament can be dissolved by a smaller bias voltage at higher temperatures, resulting in the observed tendency of the RESET voltage.

We see that the temperature variation of the forming voltage (Fig. 1b) is somewhat different from that of the SET voltage (Fig. 3a). Up to $50{ }^{\circ} \mathrm{C}$, both the forming and SET voltages increased with increased substrate temperature. However, above $50{ }^{\circ} \mathrm{C}$, the forming voltage decreased while the SET voltage continued to increase slightly. The decrease of the forming voltage at higher temperatures may suggest a larger contribution of the segmental mobility to the forming process, although this cannot be stated categorically at this stage.

It is found that the $I-V$ curves in Fig. 2 show different polarization behaviours at two substrate temperatures; almost no electric polarization at $25{ }^{\circ} \mathrm{C}$, but large electric polarization at $70{ }^{\circ} \mathrm{C}$. This behaviour is likely to be related to the $\mathrm{OFF}$ resistance and to be explained by the thermal behaviour of the Ag-PVA film. At lower temperatures, the resistance of the film is lowered by the plasticizing effect of $\mathrm{Ag}$ salt and absorbed water. In these circumstances (low OFF resistances), the electric 
polarization almost vanishes, as seen in Fig. 2a. In contrast, at higher temperatures, water evaporation takes place, which causes a decrease in the conductivity of the Ag-PVA matrix, as discussed above. This leads to high OFF resistances and large electric polarization, as seen in Fig. 2b. Further investigation is needed to elucidate and control the mechanism that determines the electric polarization and the OFF resistance.

Finally, it is necessary to emphasize that the temperature behaviour in the resistive switching of the Ag/Ag-PVA/Pt device is completely different from that observed for the $\mathrm{Ag} / \mathrm{Ag}-\mathrm{PEO} / \mathrm{Pt}$ device. In the Ag/Ag-PEO/Pt device, the SET and RESET voltages decrease in magnitude with increased temperatures, and the resistive switching changed to volatile behaviour at $70{ }^{\circ} \mathrm{C}^{15}$ In contrast, the $\mathrm{Ag} / \mathrm{Ag}-\mathrm{PVA} / \mathrm{Pt}$ device continued to exhibit nonvolatile switching under similar bias condition, even at $70{ }^{\circ} \mathrm{C}$. The larger amorphous region and the stronger interaction between hydroxyl groups and water molecules in the Ag-PVA matrix contribute to the difference in device performance.

\section{Conclusions}

In summary, stable resistive switching operation for more than $10^{2}$ continuous sweep cycles has been achieved in a temperature range of between 25 and $70{ }^{\circ} \mathrm{C}$ in a Ag/Ag-PVA/Pt atomic switch. The temperature stability seems to originate from competition between the increased segmental mobility and the decreased interaction between hydroxyl groups and water molecules, which is caused by water evaporation in the Ag-PVA matrix. The high-endurance bipolar switching with a large ON/OFF resistance ratio $\left(\sim 10^{6}\right)$ indicates that the PVA-based atomic switch has the potential for use in high-temperature switch/memory applications. There is a possibility of extending the temperature window for stable operation by selecting a more appropriate polymer material. Our results indicate the importance of the thermal property of the polymer matrix in controlling the switching characteristics of SPE-based atomic switches.

\section{Conflicts of interest}

There are no conflicts to declare.

\section{Acknowledgements}

This work was supported in part by a Grant-in-Aid for Scientific Research on Basic Research C (No. 17K05065).

\section{Notes and references}

1 T. Janoschka, M. D. Hager and U. S. Schubert, Adv. Mater., 2012, 24, 6397.

2 T. W. Kemper, R. E. Larsen and T. Gennett, J. Phys. Chem. C, 2014, 118, 17213.

3 C. Yan and P. S. Lee, Small, 2014, 10, 3443.

4 S.-T. Han, Y. Zhou and V. A. L. Roy, Adv. Mater., 2013, 25, 5425 .
5 W.-P. Lin, S.-J. Liu, T. Gong, Q. Zhao and W. Huang, Adv. Mater., 2014, 26, 570.

6 Y. Chen, G. Liu, C. Wang, W. Zhang, R.-W. Li and L. Wang, Mater. Horiz., 2014, 1, 489.

7 S. Wu, T. Tsuruoka, K. Terabe, T. Hasegawa, J. P. Hill, K. Ariga and M. Aono, Adv. Funct. Mater., 2011, 21, 93.

8 K. Krishnan, T. Tsuruoka, C. Mannequin and M. Aono, Adv. Mater., 2016, 28, 640.

9 K. Krishnan, T. Tsuruoka and M. Aono, Jpn. J. Appl. Phys., 2016, 55, 06GK02.

10 K. Terabe, T. Hasegawa, T. Nakayama and M. Aono, Nature, 2005, 433, 47.

11 T. Hasegawa, K. Terabe, T. Sakamoto and M. Aono, MRS Bull., 2009, 34, 929.

12 K. Krishnan, M. Muruganathan, T. Tsuruoka, T. Mizuta and M. Aono, Adv. Funct. Mater., 2017, 27, 1605104.

13 K. Krishnan, M. Muruganathan, T. Tsuruoka, T. Mizuta and M. Aono, Jpn. J. Appl. Phys., 2017, 56, $06 \mathrm{GF02.}$

14 K. Krishnan, M. Aono and T. Tsuruoka, Nanoscale, 2016, 8, 13976.

15 S. R. Mohapatra, T. Tsuruoka, K. Krishnan, T. Hasegawa and M. Aono, J. Mater. Chem. C, 2015, 3, 5715.

16 W. Zhang, C. Wang, G. Liu, X. Zhu, X. Chen, L. Pan, H. Tan, W. Xue, Z. Ji, J. Wang, Y. Chen and R.-W. Li, Chem. Commun., 2014, 50, 11856.

17 M. I. Baker, S. P. Walsh, Z. Schwartz, B. D. Boyan and J. Biomed, Mater. Res. Bull., 2012, 100B, 1451.

18 H. N. Chandrakala, B. Ramaraj, Shivakumaraiah, J. H. Lee and Siddaramaiah, J. Alloys Compd., 2013, 580, 392.

19 J.-M. Yang, N.-C. Wang and H.-C. Chiu, J. Membr. Sci., 2014, 457, 139.

20 M. Jiang, J. Zhu, C. Chen, Y. Lu, Y. Ge and X. Zhang, ACS Appl. Mater. Interfaces, 2016, 8, 3473.

21 C. S. Lim, K. H. Teoh, C. W. Liew and S. Ramesh, Mater. Chem. Phys., 2014, 143, 661.

22 G. Hirankumar, S. Selvasekarapandian, N. Kuwata, J. Kawamura and T. Hattori, J. Power Sources, 2005, 144, 262.

23 H. Gao and K. Lian, ACS Appl. Mater. Interfaces, 2014, 6, 464.

24 D. S. Kim, H. B. Park, J. W. Rhim and Y. M. Lee, J. Membr. Sci., 2004, 240, 37.

25 I. Valov, E. Linn, S. Tappertzhofen, S. Schmelzer, J. van den Hurk, F. Lentz and R. Waser, Nat. Commun., 2013, 4, 1771.

26 S. Tappertzhofen, I. Valov, T. Tsuruoka, T. Hasegawa, R. Waser and M. Aono, ACS Nano, 2013, 4, 6369.

27 G. Hirankumar, S. Selvasekarapandian, N. Kuwata, J. Kawamura and T. Hattorii, J. Power Sources, 2005, 144, 262.

28 B. Sarti and M. Scandola, Biomaterials, 1995, 16, 785.

29 I. Palacios, R. Castillo and R. A. Vargas, Electrochim. Acta, 2003, 48, 2195.

30 D. T. Turner and A. Schwartz, Polymer, 1985, 26, 757.

31 J. B. González-Campos, E. Prokhorov, I. C. Sanchez, J. G. Luna-Bárcenas, A. Manzano-Ramírez, J. González-Hernández, Y. López-Castro and R. E. del Río, J. Nanomater., 2012, 11, 925750.

32 R. M. Hodge, T. J. Bastow, G. H. Edwards, G. P. Simon and A. J. Hill, Macromolecules, 1996, 29, 8137. 